(c) 2010 IEEE. Personal use of this material is permitted. Permission from IEEE must be obtained for all other uses, in any current or future media, including reprinting/republishing this material for advertising or promotional purposes, creating new collective works, for resale or redistribution to servers or lists, or reuse of any copyrighted component of this work in other works. 


\title{
Teaching the Electronic Design and Embedded System Course with Body Sensor Nodes
}

\author{
Kiing-Ing Wong, Nader Barsoum, Cho Zin Myint \\ Department of Electrical and Computer Engineering \\ Curtin University of Technology \\ Miri, Sarawak, Malaysia \\ wong.kiing.ing@ curtin.edu.my
}

\begin{abstract}
The body sensor nodes armed with a MSP430 microcontroller, a IEEE 802.15.4 radio chip, a memory flash and a electronic amplifier circuits is proposed as an educational platform for electronic design and embedded system courses. The body sensor nodes are designed based on a commercial wireless sensor network (WSN) device that contains the microcontroller, radio chip and memory flash in a single platform. The WSN device also supplies the connection pins for I/O signals, ADC, SPI and UART functionalities, to control an electronic amplifier circuit. For electronic design courses, the ease of creating the body sensor node, will be hard to resist by the students. An electronic amplifier is designed and fabricated by the students in the laboratory in the electronic design courses. The WSN device is stacked on the top of the electronic amplifier circuit to prototype a new body sensor node. For the embedded system course students, the unique properties of TinyOS used as the operating system for the body sensor node allowed the students see the effects of the software in short time.
\end{abstract}

\section{WSN;TinyOS;ECG;PPG;}

\section{INTRODUCTION}

The ability to develop tiny, wearable and wireless medical sensing nodes (referred to as body sensor nodes) to replace some of the existing medical systems would have a profound impact on many aspects of clinical practice. For example, a long hour vital signs have to be captured and analysed in some of the cardio related diseases. In the current practice, the patients are required to lie still on the bed for a very long period of time, in some cases overnight in hospital for several days, in order to capture the symptom of disease. However, the body sensor nodes allow continuous vital signs to be monitored and detected while the patient is at home or even at work and away from hospital. The immediate notification of patient deterioration can be detected by analysing the data received regularly through internet or telecommunication system.

Wireless medical telemetry system is not totally new. A number of wireless medical monitors are currently on the market, including ECG, pulse oximeters, blood pressure monitors and fetal heart rate monitor [1 - 3]. These devices are wirelessly linked to allow the remote measurement and reporting of vital signs to the healthcare personal. However, the commercially available wireless/telemetry monitors are usually expensive, big in size, and only confined in hospital uses or some private patients [4]. The systems are designed only to replace the wired monitor system with wireless link using Bluetooth. Few of these systems are designed to be wearable and none are low-cost and certainly not very widely used. As an example the Welsh-Ally Micropaq Pocket PC-Based Resting ECG [3] measures over $18 \mathrm{~cm} \mathrm{x}$ $8.8 \mathrm{~cm} \mathrm{~cm} \times 4 \mathrm{~cm}$, weighs over nearly half a kilogram, is expensive and with limited usage.

A wireless sensor network (WSN) is a network consisting of spatially distributed autonomous devices, whose primary goal is to obtain miniature ultra-low-power sensor devices. The early WSN was confined to military applications such as battlefield surveillance. In recent years, it has found more applications in civilian and industrial areas, including environment and habitat monitoring, healthcare applications, home automation and industrial instrumentations. The WSN devices have became smaller, cheaper, lower power consumption and faster deployment capability. [4 - 6]. The WSN devices can be imagined as small computers that consist of a low-power microcontroller, sensor circuits, a radio transceiver and a power source. Therefore, a new wireless medical telemetry device designed based on the WSN devices inherit the characteristics, such as low-cost, low-power, light-weight, wirelessly linked and fast deployment $[7,8]$. The body sensor node is referred to the wireless medical telemetry device with the improved weight, cost and portability.

The body sensor nodes are introduced to the students in electronic design and embedded system courses. For the electronic design courses, the students are required to design an electrocardiogram (ECG) and a photoplethysmography (PPG) amplifier circuits in the laboratory sections. The students get hand-on experience in layout a printed circuit board (PCB) on a software platform, fabricating the PCB board and assembling the components on the PCB. The embedded system courses, the students are given opportunity to experience the TinyOS operating system on body sensor nodes. This paper describes procedures on setting up the laboratory sections for the students.

\section{ECG AMPLIFIER CIRCUIT DESIGN}

The electronic system design is normally fall into the following steps:

$\square$ Define functional requirements

$\square$ Develop conceptual scheme

Select and acquire components

$\square$ Develop detailed circuit

$\square$ Layout PCB

$\square$ Assemble, test and debug 
Write firmware/software

Due to limited availability of time, the functional requirements of system, conceptual design and selection of components are all specified clearly in the laboratory sheets. The students are taught about the basic of ECG signals of human body. They will understand the custom circuit used for amplifying the ECG signals. They will develop schematic and PCB layout in Eagle PCB software package in the laboratory. We selected the Eagle PCB Light Edition for the course because it is free of licensing. It is limited to board area of $1000 \times 80 \mathrm{~mm}$ size and only two signal layers design and the schematic [9]. Furthermore, Eagle PCB is also one of the most popular software for PCB layout development.

The designed PCB diagram will be fabricated using a PCB prototyping machine in the university. The PCB prototyping machine is commercially available from MITS electronic [10]. A commercial PCB prototyping machine costs at least USD 10,000, depending on the models of machine which gives different accuracy and other features, such as automatic bit change and 3 axis motor controls. We can prototype an electronic circuit and start testing within a few hours time by using the PCB prototyping machine.

Most of the integrated circuit (IC) chips are only available in Surface Mount Technique (SMT) format, and most of the through hole type IC chips are already obsolete. The SMT type IC chip can not be tested on a breadboard. Therefore, a PCB prototyping machine is needed to mill/fabricate a PCB board for testing purpose.

One laboratory section is allocated for make a schematic diagram in Eagle PCB software and created component libraries. The purpose of schematic diagram is to make easy routing in the PCB layout. In other words, the components are linked in the schematic diagram. One of the laboratory sections is to teach student how to assemble the component on the PCB board using PCB layout in Eagle PCB software.

The students are required to do some testing and debugging. The PCB board will be stacked on the bottom of a WSN device to complete the design of a body sensor node. They are not required to learn the firmware/software development in the electronic design course. In other words, the WSN device is already programmed to sample the ECG at high sampling rate (e.g. $100 \mathrm{~Hz})$.

\section{A. ECG Amplifier Circuti Design}

Almost all the ECG amplifiers are designed based on an instrumentation amplifier. The instrumentation amplifier is like an op-amp but with more stringent characteristics, such as a better CMRR (common mode rejection ratio) performance, a lower biasing current and a higher input impedance than the op-amp. The custom ECG amplifier introduced to the student is shown in Figure 1. The ECG amplifier circuit is operated by 3 volt DC supply voltage, and it has four steps:

1) Limited input gain of instrumentation amplifier (i.e.Gain= 5).

2) Implement high-pass filtering at the cut-off frequency of $0.05 \mathrm{~Hz}$ to remove the common signals.

3) Implement low-pass filtering (or anti-aliasing

4) filtering) in the output stage at the cut-off frequency
5) of $106 \mathrm{~Hz}$; and

6) Apply high gain in the output stage to boot the ac

7) signal by 200 times.

\section{B. ECG Amplifier Circuit Experimental Testing}

An ECG sensor node that consisted of an amplifier circuit and a general purpose WSN platform. The ECG amplifier circuit is designed on a $30 \mathrm{~mm}$ x $40 \mathrm{~mm}$ size PCB board, so that it can be attached to the WSN platform for data acquisition. The circuit uses SMT parts, including a Texas Instrument (TI) INA126 instrument amplifier, an OPA4132 op-amp package and the 1206 format chip resistors and capacitors, in order to minimize to the desired size. All the components are packed into a $3 \mathrm{~cm} \mathrm{x} 4 \mathrm{~cm}$ board size.

Figure 2 shows the experimental setup for the testing the ECG amplifier circuit. A patient simulator is used to generate simulated ECG signals from human skin. The magnitude and the heart beat rate of the patient simulator are adjustable, ranging from 0.1 millivolts to 5 millivolts and $60 \mathrm{BPM}$ to $200 \mathrm{BPM}$, respectively. The final experimental set up for the ECG sensor node is shown in Figure 3. The ECG body sensor node is tied to V2 and V4 output pins of the patient simulator. The amplified ECG signal is sampled and processed by an ECG body sensor node. The resultant signal is radioed from the ECG body sensor node to a personal computer (PC) through a basestation node for displaying and storage. The base station node receives the messages from the body sensor node and forwards the message to the PC through the serial port.

\section{PPG AMPLIFIER CIRCUIT DESIGN}

The detection of PPG signals can be divided into two modes: transmittance and reflectance [11, 12]. In the transmittance mode, a light source is emitted in to the tissue and a light detector is placed in the opposite side of the

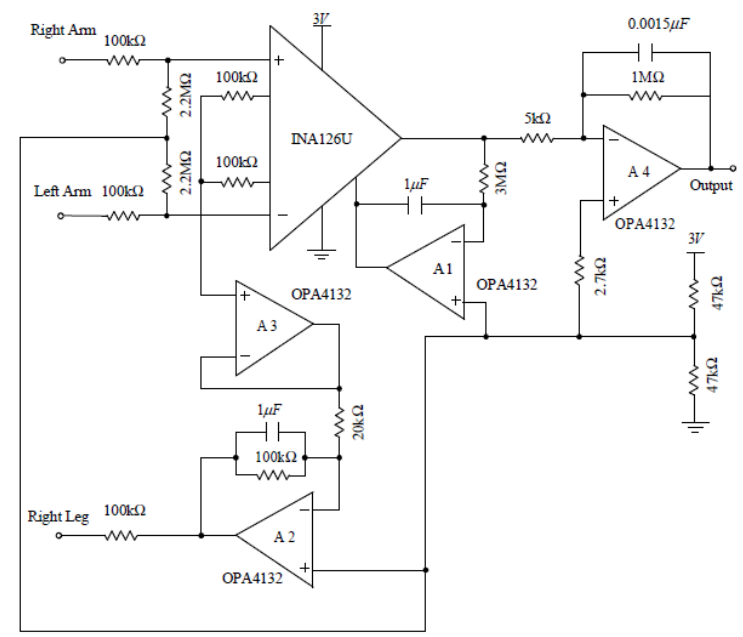

Fig. 1. ECG amplifier circuit. 


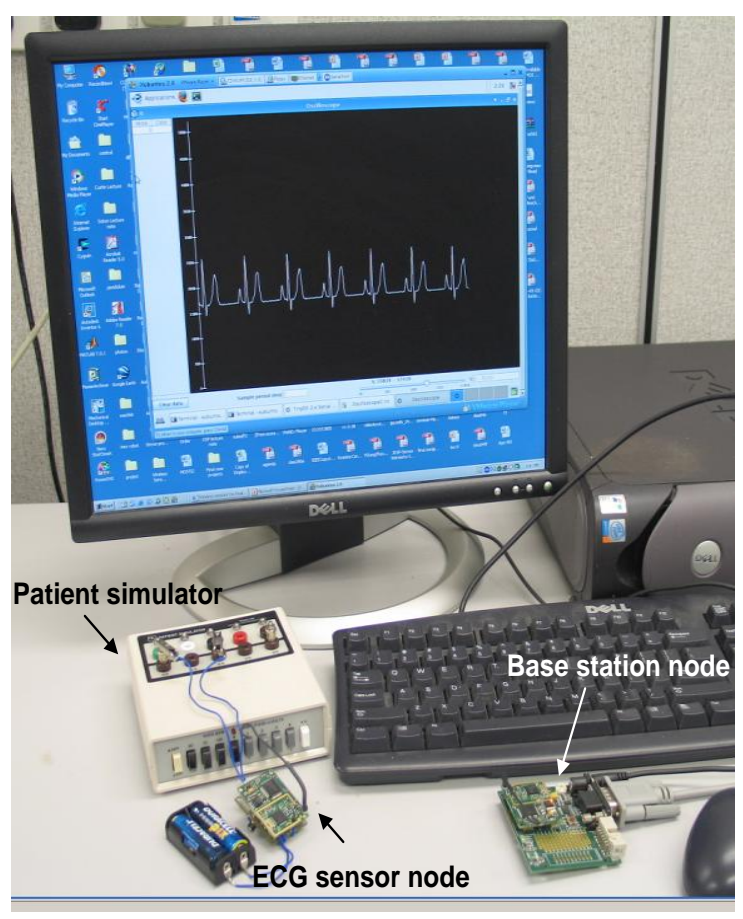

Fig. 2. Experimental setup consisted of a patient simulator, a ECG sensor node a base-station node and a PC.

tissue to measure the resultant light. The transmittance PPG mode is applied to a restricted body parts, such as finger and ear lobe. In the reflectance mode, the light source and the light detector are place on the same side of the medium. The light is emitted into the tissue and the reflected light is measured by the light detector. Therefore, the reflectance PPG sensor can be applied to any parts of human body. This method saves more power than the transmittance one, since it does not require as much light intensity as the transmittance one, in order to get a readable data. A reflectance PPG sensor incorporated into helmet can provide the physiological status of soldiers on the battlefield and the personals working in the hazardous environment.

\section{A. PPG Amplifier Circuti Design}

Figure 3 presents the PPG sensor circuit diagram. It consisted of a PPG amplifier circuit and a WSN node. Both are connected to a single 3 volt DC power supply. The $\mathrm{ADC} 0$ and $\mathrm{ADC} 1, \mathrm{DAC} 0$ and $\mathrm{I} / \mathrm{O}$ ports are wired to the WSN node for controlling. The I/O port of WSN node is driven by the WSN node to turn on the infrared LED for PPG signal reading and turn off immediately after reading. The DAC controls the current and thus the light intensity of the LED.

The OPT101 chip generates a voltage signal from the received light. The voltage signal consists of a large DC component superimposed on a small AC component. The AC component magnitude is between 0.1 and $1 \%$ of the DC component. In PPG detection, DC component is filtered by using either a digital filter [7] or an analogue filter [8]. Due

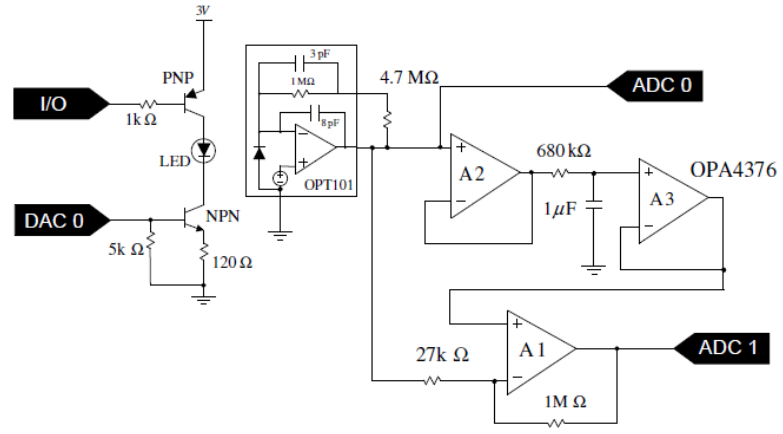

Fig. 3. ECG amplifier circuit.

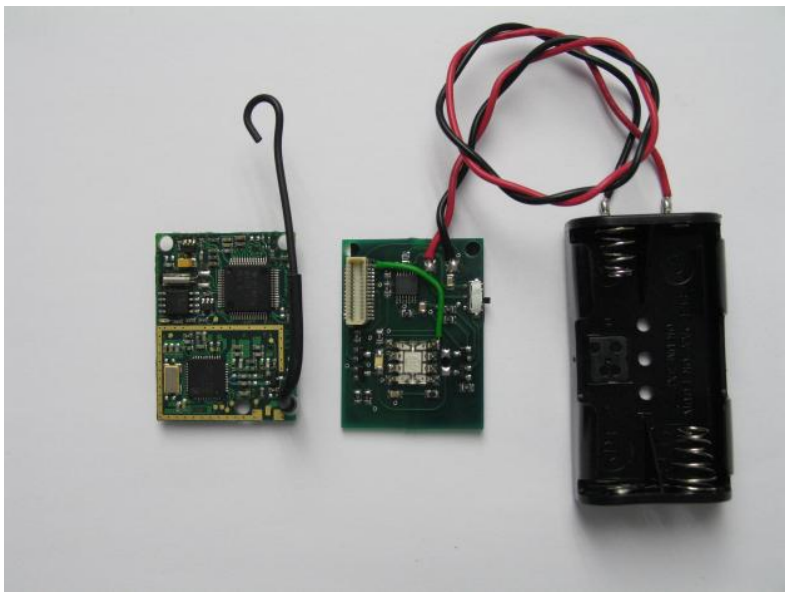

Fig. 4. A reflectance PPG sensor node consisted of a WSN node (left), an amplifier circuit board (middle) and a battery case.

to the limitation of the computation power of the WSN node, the analogue filter is used in the design here.

The analogue filter consisted of two op-amps (operational amplifiers): A2 and A3 (shown in Figure 2 (b)) [6]. The A2 op-amp is used to buffer the PPG signals into a RC low-pass filter that has a cut-off frequency of $0.23 \mathrm{~Hz}$. The output signals are largely DC signals which is fed into the non-inverting input of the A1 differential op-amp circuit via the A3 op-amp. The A3 op-amp is acted as the buffer for the RC low-pass filter and the A1 differential amplifier circuit. The differential amplifier circuit will attenuate all the DC signals and amplify only the AC signals as the input PPG signals to the WSN node. The differential amplifier circuit has a voltage gain of $37 \mathrm{~V} / \mathrm{V}$.

The experimental setup of PPG system consisted of a PPG body sensor node and a base station (which is the similar to the one used for the ECG body sensor node experiment as shown in Figure 3). Figure 5 shows the PC display of the raw PPG signals from a finger of a student. The PPG signals are sampled at $200 \mathrm{~Hz}$ rate, with a 12 bits resolution.

\section{TINYOS}

In the embedded system courses, the students are introduced to TinyOS operating system. TinyOS that is a small, open source, energy efficient, software operating 


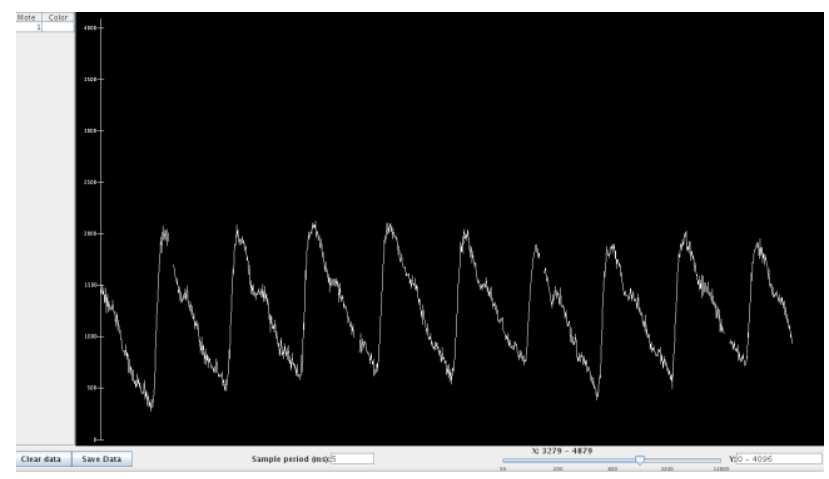

Fig. 5. PC display of raw PPG signals of finger.

system first developed by UC Berkeley, USA [14]. It features a component-based architecture to promote code reuse and enables rapid implementation. TinyOS components include data acquisition, flash memory access, wireless communication protocols, and task scheduling. Most of the components are configured from low level components to reflect its functionality clearly by hiding the hardware setting. The application designers allow customizing their applications by choosing the essential components, therefore achieve minimum code size.

TinyOS-based application is built on a nesC dialect using the components that follows the split-phase operations [14]. Like hardware operations, a TinyOS components command (or signal) a function is non-blocking. The TinyOS component issues an event (or callback), at some point later, after completion of the function of component.

\section{A. Wireless Sensor Network Device}

The wireless sensor network (WSN) device, namely TinyNode 584 [11], is used to examine TinyOS in the laboratory. TinyNode 584 is obtained from Shockfish SA, Switzerland that has the resemblance of the other commercially available WSN devices, such as TelosB and Imote 2 from Crossbow [9]. These WSN nodes are designed based on low-power microcontroller, and have an on-board flash memory, a radio transceiver unit and an expansion connector. The expansion connector is used to control the external electronic circuit, usually the sensors. Figure 6 shows the block diagram of a general WSN device.

TinyNode 584 has all the components packed into a PCB board of $40 \times 30 \mathrm{~mm}$ size. TinyNode 584 utilizes the 868 $\mathrm{MHz}$ industrial, scientific and medical (ISM) band for unlicensed operation. This sub-GHz ISM bands has better penetration, resulting in better range compared to $2.4 \mathrm{GHz}$ WSN nodes. TinyNode 584 offers communication ranges of 1800 meter and exceeds current WSN nodes by a factor of 3 to 5, while consuming similar energy [13].

We avoided using the TinyNode 184 which is released later than TinyNode 584. This is because TinyNode 184 uses MSP430F2417 microcontroller which does not have any onchip digital-to-analog-converter (DAC). To generate analog waveform will require additional circuit and may complicate

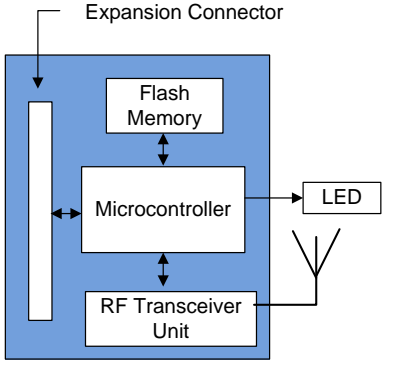

Fig. 6. Block diagram of wireless sensor network device.

the design. Most microcontrollers of WSN nodes integrate some basic ADC, but some do not include DAC.

\section{B. TinyOS tutorials}

Three laboratory sections are allocated to explain simple TinyOS operations to the students. In the first laboratory section, students learn how to install the latest version of TinyOS (TinyOS 2.1.1) into PC. Then, they will start experiencing timer and LED modules of TinyOS. They will program the WSN device to blink the LEDs at specified rate by controlling the timer and output pins.

In the second and third laboratory sections, student will experiencing modules to do sampling analog signals, storing digital data, transmitting digital data to base station and collecting data from network and display on PC.

In the fourth laboratory sections, students are required to develop a TinyOS based firmware to control the PPG body sensor node. The PPG body sensor node requires more complicated controls than ECG body sensor node. The PPG body sensor node requires the light intensity to be sampled for and controlling the light intensity, using the digital to analog (DAC) module.

Figure 7 shows the interrupt service routine of the light intensity sampling. The application program checks whether the light intensity is within the target brightness (since ADC0 voltage signals are consisted of DC component predominantly). If the LED is outside the target brightness, the application program tries to bring it back to the right direction. The DAC range is renewed subsequently with the new LED intensity level.

Figure 8 shows the oscilloscope display of electric current values of PPG based body sensor node. There are two large current peaks that are resulted from the wireless communication operations. There are nine smaller peaks in between two large current peaks. It shows that the LED is turned on for reading, and it is turned off after reading (in order to save energy consumption of body sensor node. A total of nine PPG sample data are stored before it is radio to base station. The current information also shows that the program latency is not an issue, the program is given sufficient time to recover at each operation.

\section{CONCLUSION}

The old way of teaching may not be effective any more. The students were expected to receive anything and 


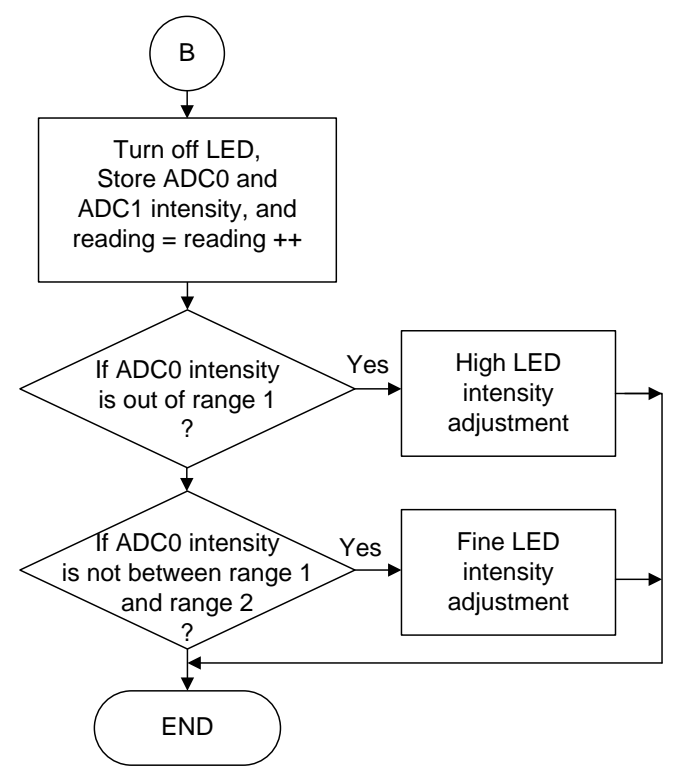

Fig. 7. Interrupt service routine used to control the LED light intensity.

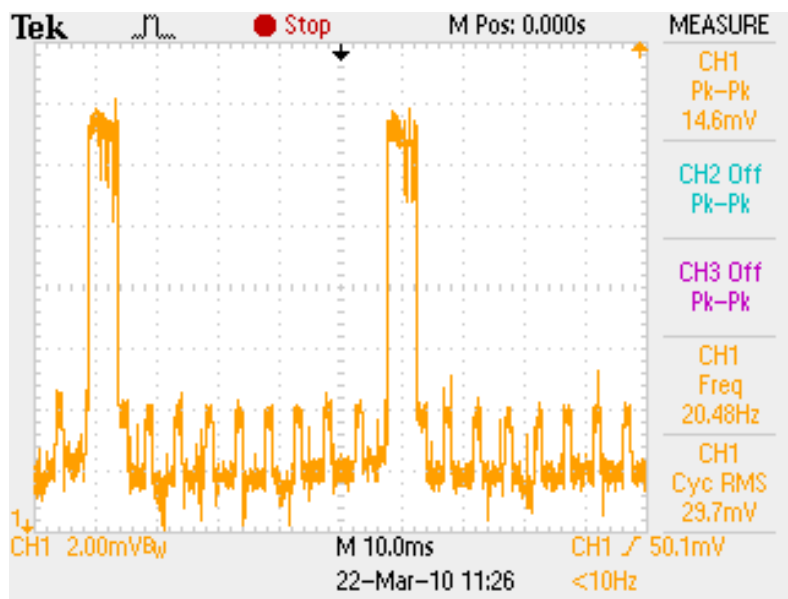

Fig. 8. The oscilloscope display of electric current values of PPG based body sensor node.

everything delivered by the professor. They were responsible to come to lecture and get the information. Those who do not come to the lecture often will expect not to do very well in their final examination. The professor often covered a lot of theoretical concepts and analytical skills for a simple electronic circuit. It is also true for most of the electronic design text books used in most of the university.

The students often found the electronic subject uninteresting because they can not find any usefulness of the electronic circuits that they learned. They often complain that the outcomes of the subject are not relevant to their future employment. Their criticism might be acceptable because the electronic circuits are often too simple to build any useful electronic device. In real-word practice, skills of prototyping the electronic systems within a limited time frame is import that is often not covered in the electronic design courses. We introduce the body sensor node to the student in laboratory. Students have the hand-on experience on how to prototype an ECG amplifier, and then attach the ECG amplifier on a WSN device to realized a new ECG based body sensor node.

Embedded systems are integrations of electronic hardware and software. A body sensor node is a rich hardware platform for studying the embedded system. It consisted of a microcontroller, a radio transceiver chip, a memory flash and an electronic amplifier circuit. The application software designed based on TinyOS contain a standard and unique structure. Its program code is small and highly efficient, and therefore suitable for operate an embedded system, such as the body sensor node. The students of embedded system courses are able to jump-start on testing various hardware functionalities, such as I/O port, ADC, DAC and Serial Peripheral Interface (SPI), and wireless communication protocols. They are able to investigate the high level concepts and start writing the applications for the PPG based body sensor node.

\section{ACKNOWLEDGMENT}

This work is partly funded by the Malaysian Ministry of Science, Technology and Innovation (MOSTI) under Project No. : 01-02-07-SF0010. The author would like to thank the university for the conference attendance supports.

\section{REFERENCES}

[1] GMP Wireless Medicine, Inc. LifeSyn Wireless EKG system, http://www.wirelessecg.coin/

[2] HealthFrontier, Inc. ecgAnywhere, http://www.healthfrontier.com

[3] Welch Ally, Inc. Micropaq Wireless Patient Monitor, http://www.monitroing.welchallyn.com/products/wireless/

[4] V. Shnnayder, B. R. Chen, K. Lorinez, R. F. Thaddeus, "Sensor network for medical care," Technical Report TR-08-05, Division of Engineering and Applied Sciences, Harvard University 2005.

[5] B. Lo and G. Z. Yang, "Architecture for body sensor networks," IEE Proceeding ofPerspective in Pervasive Computing, pp 23-28, 2006.

[6] R. F. Thaddeus, G. Y. Wei and M. Welsh, "A portable, low-power, wireless two-lead EKG system," IEEE Proceedings of the 26h Annual International Conference of the Medicine and Biology Society, pp 2141- 2144, 2004.

[7] K. I. Wong, "Rapid prototyping of a low-power, wireless, reflectance photoplethysmography system", Accepted for publication in the IEEE proceeding of the $8^{\text {th }}$ International Workshop on Wearable and Implantable Body Sensor Network, Biopolis, Singapore, 2010.

[8] K. I. Wong and Mel M. S. Ho, "Wearable biosignal monitoring nodes for real-time electrocardiogram and motion measurement", IEEE Proceeding of the $5^{\text {th }}$ International Workshop on Wearable and Implantable Body Sensor Network, Hong Kong, page190-193, 2008.

[9] Eagle PCB: http://www.cadsoft.de/

[10] MITS: http://www.mitspcb.com/edoc/epri.htm

[11] Chan V., Underwood S., "A single-chip pulsoximeter design using the MSP430", in Texas Instruments Application Notes, 2005.

[12] Honghui Jiang, "Motion-artifact resistant design of photoplethysmograph ring sensor for driver monitoring", Master of Science dissertation, Dept. Mech. Eng., M.I.T, 2003.

[13] Crossbow webpage http://www.xbow.com

[14] TinyOS http://www.tinyos.net 